\title{
Terahertz photoconductivity and plasmon modes in double-quantum-well field-effect transistors
}

\author{
X. G. Peralta ${ }^{a}$ and S. J. Allen \\ Center for Terahertz Science and Technology, University of California, Santa Barbara, California 93106 \\ M. C. Wanke, N. E. Harff, J. A. Simmons, M. P. Lilly, and J. L. Reno \\ Sandia National Laboratories, Albuquerque, New Mexico 87185 \\ P. J. Burke and J. P. Eisenstein \\ Department of Physics, California Institute of Technology, Pasadena, California 91125
}

(Received 18 February 2002; accepted for publication 5 June 2002)

Double-quantum-well field-effect transistors with a grating gate exhibit a sharply resonant, voltage tuned terahertz photoconductivity. The voltage tuned resonance is determined by the plasma oscillations of the composite structure. The resonant photoconductivity requires a double-quantum well but the mechanism whereby plasma oscillations produce changes in device conductance is not understood. The phenomenon is potentially important for fast, tunable terahertz detectors. (c) 2002 American Institute of Physics. [DOI: 10.1063/1.1497433]

Double-quantum-well (DQW) heterostructures are important in the scientific exploration of correlated electron states in two-dimensional electron systems ${ }^{1}$ and potentially important for novel field-effect transistors that add functionality by controlling electron transfer between the quantum wells. ${ }^{2}$ Interwell transfer can also be promoted by terahertz photon assisted tunneling, opening the possibility of fast, voltage tunable terahertz $(\mathrm{THz})$ detectors. ${ }^{3}$ This motivated our research on $\mathrm{THz}$ response of DQW field-effect transistors (FETs). We report the THz photoconductivity of DQW FETs in which the gate is a periodic metallic grating. Strong photoresponse occurs at the plasma resonance of the composite structure. Other detector proposals make use of plasmon modes in single two-dimensional electron gas (2DEG) systems. ${ }^{4}$ But, the relatively strong resonant response that we report here appears to require the presence of a DQW. We model the resonant response with a transmission line model of the collective modes of the 2DEGs and correlate the observed resonances with standing plasmon resonances under the metallic part of the grating gate. While the work was motivated by the concept of interwell transfer, the actual mechanism that gives rise to this response is not understood.

The FETs are fabricated from modulation doped GaAs/ AlGaAs DQW heterostructures grown by molecular beam epitaxy. Both wells are $200 \AA$ wide and are separated by a 70 $\AA$ barrier. The nominal electron densities are $n_{\text {upper }}=1.7$ $\times 10^{11} \mathrm{~cm}^{-2}$ and $n_{\text {lower }}=2.57 \times 10^{11} \mathrm{~cm}^{-2}$ : the $4.2 \mathrm{~K}$ mobility is $\sim 1.7 \times 10^{6} \mathrm{~cm}^{2} / \mathrm{V} \mathrm{s}$. A $2 \times 2 \mathrm{~mm}$ mesa is defined and ohmic contacts to both quantum wells form source and drain. A $700-\AA$-thick TiAu grating gate (with no metallization between the grating fingers) is evaporated with the lines of the grating perpendicular to the current flow. We explored 4 and $8 \mu \mathrm{m}$ periods; half the period is metal. The grating modulates the electron density when a voltage is applied, selects wave vectors of the excited plasmon and, coincidentally, produces both normal and transverse $\mathrm{THz}$ electric fields. See

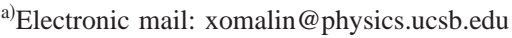

inset in Fig. 1 for a cross section of the sample.

We apply a constant source-drain current of $100 \mu \mathrm{A}$, focus the radiation onto the sample, and study the photoconductive response of the DQW as a function of gate voltage, $\mathrm{THz}$ frequency, and temperature. The radiation sources are the free-electron lasers at the University of California, Santa Barbara, which cover a frequency range between $120 \mathrm{GHz}$ and $4.8 \mathrm{THz}$. The response is linear with respect to sourcedrain current (photoconductive, not photovoltaic) and with respect to incident power.

Figure 1 shows the conductance of the DQW channels as a function of gate voltage (depletion curve) and the photoconductive response at four different temperatures at a frequency of $570 \mathrm{GHz}$ for the $4 \mu \mathrm{m}$ grating period sample. The depletion curve shows that at $V_{g} \sim-0.80 \mathrm{~V}$ the top 2DEG is fully depleted under the metallic portion of the grating gate, forming an array of disconnected $2 \mu \mathrm{m}$ stripes. As the gate voltage becomes more negative, the lower 2DEG is also patterned into stripes until the conductance goes to zero when both layers are cut off from the source and drain.

At $T=2.2 \mathrm{~K}$ the photoresponse at $570 \mathrm{GHz}$ shows a broad structured peak at $V_{g} \sim-1.19 \mathrm{~V}$. This feature increases and narrows as a function of increasing temperature

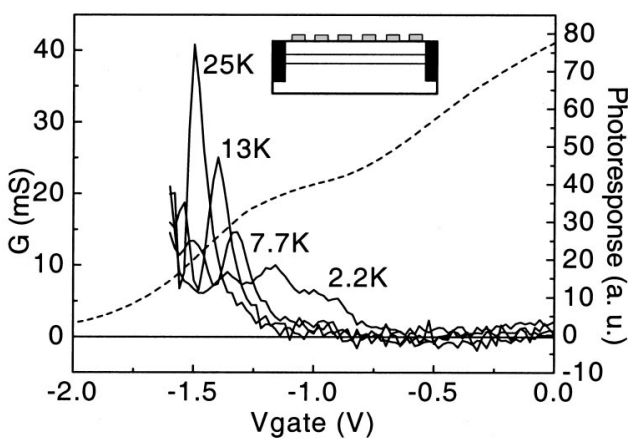

FIG. 1. Source-drain conductance as a function of gate voltage (dashed). Terahertz photoresponse at $570 \mathrm{GHz}$ at four different temperatures (solid). Grating period is $4 \mu \mathrm{m}$. Schematic cross section of the devices (inset). 


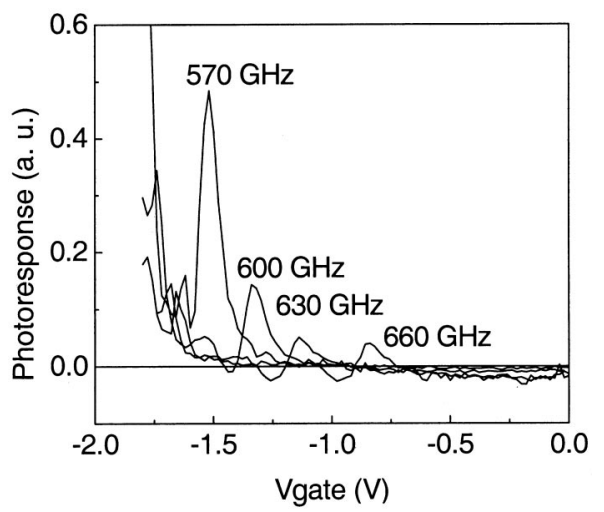

FIG. 2. Terahertz photoresponse as a function of gate voltage at four different frequencies. The temperature was $T=25 \mathrm{~K}$ and the source-drain current was $I_{\mathrm{SD}}=100 \mu \mathrm{A}$. Grating period is $4 \mu \mathrm{m}$.

up to around $T=40 \mathrm{~K}$, after which it decreases. The temperature dependence is unexplained at this time but enhances the potential usefulness as a future detector.

Figure 2 shows the gate dependent photoresponse at $T$ $=25 \mathrm{~K}$ for different radiation frequencies. The resonant peak moves to less negative gate voltage, higher electron density, as we increase the frequency of the incident radiation; this is expected plasmon behavior.

Figure 3 shows the photoresponse as a function of gate voltage at $600 \mathrm{GHz}$ and $T=25 \mathrm{~K}$ for the 4 and the $8 \mu \mathrm{m}$ periods. There are multiple resonances in the photoresponse of the $8 \mu \mathrm{m}$ period which are the high order spatial harmonics (see below). The presence of many higher harmonics indicates that the $\mathrm{THz}$ electric field exciting the plasmons is very nonuniform, possibly localized at the edges of the metal.

Plasmon modes have been studied both theoretically, and experimentally, ${ }^{7-10}$ and there have been many different approaches to modeling them. ${ }^{11,12}$ The structure presented in this letter is complicated and can be split into two regions. ${ }^{13,14}$ there is a double layer ungated region and a double layer gated region with a varying electron density that becomes a single layer at large negative gate voltages. We chose to model the collective response of the composite structure by treating the DQW as a single one, ignoring the effect of fringing fields on the ungated regions and using an equivalent circuit (lower inset in Fig. 4). In the ungated region we combine the sheet densities of the two quantum

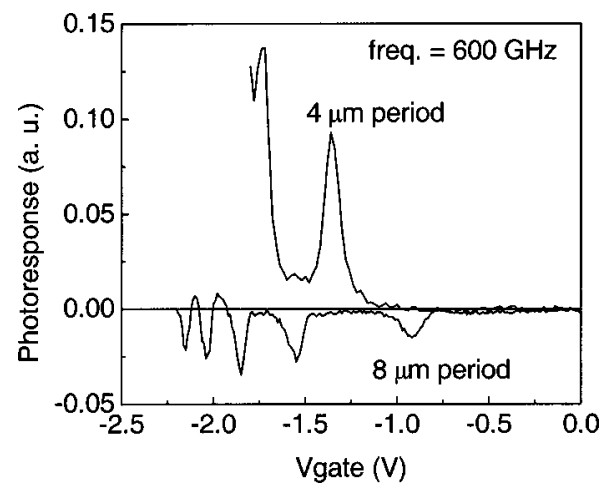

FIG. 3. Terahertz photoresponse at $600 \mathrm{GHz}$ for two samples with different grating periods: 4 and $8 \mu \mathrm{m}$ at $T=25 \mathrm{~K}$. Observe the multiple resonances in the $8 \mu \mathrm{m}$ period sample.

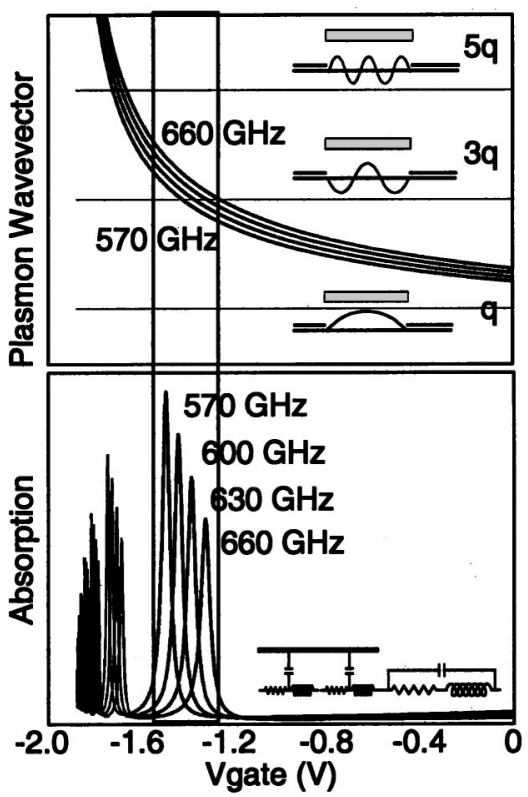

FIG. 4. Top: Plasmon wave vector as a function of gate voltage, with frequency as a parameter. Horizontal lines: odd number of plasmon $1 / 2$ wavelength underneath the metal (inset). Bottom: absorption as a function of gate voltage for the frequencies used in the experiment. Elements of the transmission line model (inset).

wells into one ( $\left.n_{\text {effective }}=n_{\text {upper }}+n_{\text {lower }}\right)$ and keep it fixed. The response for the gated region involves standing waves under the grating metal. These are modeled by a "transmission line" with a variable density 2DEG (ranging from $n_{\text {effective }}$ to zero).

The total impedance is the sum of the impedances of the gated and ungated regions (in series). We calculate the ratio of the absorbed power to the incident power as a function of the electron density under the grating metallization. The lower plot in Fig. 4 shows the resulting normalized absorption as a function of gate voltage for the $4 \mu \mathrm{m}$ period at various frequencies. The resonant peak moves to lower gate voltage as we increase the frequency of the radiation.

The upper plot helps us develop some intuition by showing the plasmon wave vector in a uniformly metallized 2DEG at a particular frequency as a function of gate voltage. The horizontal lines correspond to an odd integer number plasmon $1 / 2$ wavelengths underneath the $2 \mu \mathrm{m}$ metal finger in our device (odd spatial harmonics). The first set of resonances in the normalized absorption (from right to left) corresponds to the plasmon with wave vector $3 q$ $=3 *(2 \pi / 4 \mu \mathrm{m})$, as indicated by the parallel vertical lines. The insets on the top right are schematic representations of the current density distribution under the metal gate for the corresponding resonant modes.

By lumping the two wells together we ignore the acoustic plasmons in the double well regions; we assume only the optical plasmon is important. Nonetheless, the model captures the dependence on frequency, period, and density and leaves little doubt that the resonance is semiquantitatively described by the collective modes. While we understand that the tunable resonance is caused by the composite plasma oscillations, the mechanism that gives rise to the change in conductance at resonance is not clear. Relevant observations follow.

to AIP license or copyright, see http://apl.aip.org/apl/copyright.jsp 
(1) While it is clear that the electron density under the gated region controls the collective plasma oscillations of the composite structure, the changes induced in the dc conductance can take place in either the gated or ungated regions.

(2) A single 2DEG processed in an identical manner showed no resonant response; the double well is necessary.

(3) The change in sign in Fig. 3 challenges the idea that interwell transfer is responsible for this response because it is hard to imagine a reversal in the direction of the electron transfer process.

(4) Coulomb interactions between two quantum wells are mediated by charge fluctuations, plasmons. ${ }^{15}$ Radiation driven charge density fluctuations can lead to enhanced scattering between electrons in the two wells.

Figure 2 shows that this device could be used as a tunable detector so we made an estimate of its noise equivalent power (NEP) without including optical coupling and found a $\mathrm{NEP}=6 \mu \mathrm{W} / \mathrm{Hz}^{1 / 2}$ and a responsivity of $R=890 \mu \mathrm{V} / \mathrm{W}$. This is not competitive with good incoherent detectors, on the other hand, the response time has been measured to be no slower than $700 \mathrm{~ns}$. If the speed is sufficiently fast, it may find use as a $\mathrm{THz}$ heterodyne detector integrated with IF (intermediate frequency) electronics.

We have demonstrated that a double-quantum-well fieldeffect transistor with a grating gate exhibits striking voltage tuned resonant photoconductive response at terahertz frequencies directly related to plasma oscillations of this composite structure. To shed light on the photoconductive mechanism, future work will explore the effects of both normal and in plane magnetic field. Optimization of the device for a tuned incoherent or coherent detector with integrated signal processing electronics is also indicated.

The authors would like to thank D. Enyeart and G. Ramian at the Center for Terahertz Science and Technology and W. Baca at Sandia National Labs. This work was sup- ported by the ONR MFEL program, the DARPA/ONR THz Technology, Sensing and Satellite Communications Program, and the ARO, Science and Technology of Nano/Molecular Electronics: Theory, Simulation, and Experimental Characterization. Sandia is a multiprogram laboratory operated by Sandia Corporation, a Lockheed Martin Company, for the United States Department of Energy under Contract No. DEAC04-94AL85000.

${ }^{1}$ J. P. Eisenstein, L. N. Pfeiffer, and K. W. West, Appl. Phys. Lett. 58, 1497 (1991).

${ }^{2}$ J. A. Simmons, M. A. Blount, J. S. Moon, S. K. Lyo, W. E. Baca, and M. J. Hafich, J. Appl. Phys. 84, 5626 (1998); J. S. Moon, J. A. Simmons, M. A. Blount, W. E. Baca, J. L. Reno, and M. J. Hafich, Electron. Lett. 34, 921 (1998).

${ }^{3}$ B. J. Keay, S. Zeuner, S. J. Allen, K. D. Maranowski, A. C. Gossard, U. Bhattacharya, and M. J. W. Rodwell, Phys. Rev. Lett. 75, 4102 (1995); H. Drexler, J. S. Scott, S. J. Allen, K. L. Campman, and A. C. Gossard, Appl. Phys. Lett. 67, 2816 (1995).

${ }^{4}$ M. S. Shur and J.-Q. Lu, IEEE Trans. Microwave Theory Tech. 48, 750 (2000); V. Ryzhii, I. Khmyrova, and M. Shur, J. Appl. Phys. 88, 2868 (2000).

${ }^{5}$ S. Das Sarma and A. Madhukar, Phys. Rev. B 23, 805 (1981).

${ }^{6}$ G. E. Santoro and G. F. Giuliani, Phys. Rev. B 37, 937 (1981).

${ }^{7}$ N. P. R. Hill, J. T. Nicholls, E. H. Linfield, M. Pepper, D. A. Ritchie, G. A. C. Jones, B. Y.-K. Hu, and K. Flensberg, Phys. Rev. Lett. 78, 2204 (1997).

${ }^{8}$ A. S. Bhatti, D. Richards, H. P. Hughes, E. H. Linfield, D. A. Ritchie, G. A. C. Jones, in Proceedings of 23rd International Conference on the Physics of Semiconductors, edited by M. Scheffler and R. Zimmerman (World Scientific, Singapore, 1996), p. 1899.

${ }^{9}$ D. S. Kainth, D. Richards, A. S. Bhatti, H. P. Hughes, M. Y. Simmons, E. H. Linfield, and D. A. Ritchie, Phys. Rev. B 59, 2095 (1999).

${ }^{10}$ D. S. Kainth, D. Richards, H. P. Hughes, M. Y. Simmons, and D. A. Ritchie, Phys. Rev. B 57, R2065 (1998).

${ }^{11}$ M. V. Krasheninnikov and A. V. Chaplik, Sov. Phys. Semicond. 15, 19 (1981).

${ }^{12}$ U. Mackens, D. Heitmann, L. Prager, J. P. Kotthaus, and W. Beinvogl, Phys. Rev. Lett. 53, 1485 (1984).

${ }^{13}$ P. J. Burke, I. B. Spielman, J. P. Eisenstein, L. N. Pfeiffer, and K. W. West, Appl. Phys. Lett. 76, 745 (2000).

${ }^{14} \mathrm{P}$. J. Burke and J. P. Eisenstein (private communication).

${ }^{15}$ H. Noh, S. Zelakiewicz, X. G. Feng, T. J. Gramila, L. N. Pfeiffer, and K. W. West, Phys. Rev. B 58, 12621 (1998). 